\title{
Histone deacetylase inhibitors suppress RSV infection and alleviate virus-induced airway inflammation
}

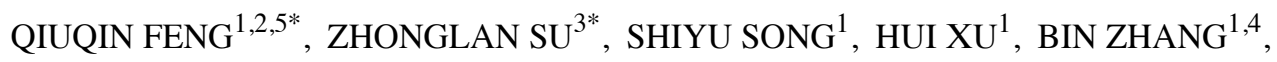 \\ LONG YI $^{1}$, MAN TIAN $^{2}$ and HONGWEI WANG ${ }^{1}$
}

${ }^{1}$ Center for Translational Medicine and Jiangsu Key Laboratory of Molecular Medicine, Medical School of Nanjing University, Nanjing, Jiangsu 210093; ${ }^{2}$ Department of Respiratory Medicine, Nanjing Children's Hospital Affiliated with Nanjing Medical University, Nanjing, Jiangsu 210008; ${ }^{3}$ Department of Dermatology, The First Affiliated Hospital of Nanjing Medical University, Nanjing, Jiangsu 210029; ${ }^{4}$ Central Laboratory, Nanjing Chest Hospital, Medical School of Southeast University, Nanjing, Jiangsu 210029; ${ }^{5}$ Zhuhai Maternity and Child Health Hospital, Zhuhai, Guangdong 519001, P.R. China

Received December 22, 2015; Accepted July 5, 2016

DOI: $10.3892 / \mathrm{ijmm} .2016 .2691$

\begin{abstract}
Respiratory syncytial virus (RSV) is the leading cause of lower respiratory tract infections in infants and young children. However, the majority of RSV-infected patients only show mild symptoms. Different severities of infection and responses among the RSV-infected population indicate that epigenetic regulation as well as personal genetic background may affect RSV infectivity. Histone deacetylase (HDAC) is an important epigenetic regulator in lung diseases. The present study aimed to explore the possible connection between HDAC expression and RSV-induced lung inflammation. To address this question, RSV-infected airway epithelial cells (BEAS-2B) were prepared and a mouse model of RSV infection was established, and then treated with various concentrations of HDAC inhibitors (HDACis), namely trichostatin A (TSA) and suberoylanilide hydroxamic acid (SAHA).
\end{abstract}

Correspondence to: Dr Hongwei Wang, Center for Translational Medicine and Jiangsu Key Laboratory of Molecular Medicine, Medical School of Nanjing University, Nanjing, Jiangsu 210093, P.R. China

E-mail: hwang@nju.edu.cn

DrManTian,Department of Respiratory Medicine,Nanjing Children's Hospital Affiliated with Nanjing Medical University, Nanjing, Jiangsu 210008, P.R. China

E-mail: tmsweet@163.com

${ }^{*}$ Contributed equally

Abbreviations: HDACis, histone deacetylase inhibitors; RSV, respiratory syncytial virus; AEC, airway epithelial cell; BALF, bronchoalveolar lavage fluid; MDA, malondialdehyde; FACS, fluorescence-activated cell sorting; NO, nitrogen monoxide; MOI, multiplicity of infection; ROS, reactive oxygen species; HCMV, human cytomegalovirus

Key words: respiratory syncytial virus, histone deacetylase, inflammation, oxidative stress
Viral replication and markers of virus-induced airway inflammation or oxidative stress were assessed. The activation of the nuclear factor- $\kappa \mathrm{B}(\mathrm{NF}-\kappa \mathrm{B})$, cyclo-oxygenase-2 (COX-2), mitogen-activated protein kinase (MAPK) and signal transducer and activator of transcription 3 (STAT3) signaling pathways was evaluated by western blot analysis. Our results showed that RSV infection in airway epithelial cells (AECs) significantly decreased histone acetylation levels by altering HDAC2 expression. The treatment of RSV-infected AECs with HDACis significantly restricted RSV replication by upregulating the interferon- $\alpha($ IFN- $\alpha)$ related signaling pathways. The treatment of RSV-infected AECs with HDACis also significantly inhibited RSV-induced pro-inflammatory cytokine release [interleukin (IL)-6 and IL-8] and oxidative stress-related molecule production [malondialdehyde (MDA), and nitrogen monoxide (NO)]. The activation of $\mathrm{NF}-\kappa \mathrm{B}$, COX-2, MAPK and Stat3, which orchestrate pro-inflammatory gene expression and oxidative stress injury, was also significantly inhibited. Our in vivo study using a mouse model of RSV infection validated these results. Treatment with HDACis alleviated airway inflammation and reduced in vivo $\mathrm{RSV}$ replication. Our data demonstrated that RSV reduced histone acetylation by enhancing HDAC2 expression. Treatment with HDACis (TSA/SAHA) significantly inhibited RSV replication and decreased RSV-induced airway inflammation and oxidative stress. Therefore, the inhibition of HDACs represents a novel therapeutic approach in modulating RSV-induced lung disease.

\section{Introduction}

Human respiratory syncytial virus (RSV), a negative-sense RNA virus belonging to the Paramyxoviridae family, is the leading cause of epidemic bronchiolitis and pneumonia in children. Given that no effective vaccines are currently available, infection with this ubiquitous virus has been associated with $40-60 \%$ of bronchiolitis cases and $15-25 \%$ of pneumonia cases in hospitalized children (1). Severe RSV infection in 
infants is associated with substantial morbidity worldwide, and previous findings have shown that RSV infection in early life increases the risk of asthma (2). Therefore, the prevention of RSV disease is of great importance.

In addition to direct epithelial damage, RSV infection in infants and immunocompromised patients typically causes severe airway inflammation, which involves the release of multiple pro-inflammatory mediators as well as inflammatory cell recruitment in peribronchial and perivascular spaces, and increased mucus production; therefore, the inhibition of viral replication and the reduction of virus-induced airway inflammation are suitable therapeutic targets in severe RSV infection (3).

Protein acetylation, an important epigenetic modification pattern, plays a key role in host defenses against viral infections. Histone deacetylases (HDACs) are enzymes that remove the acetyl moiety from specific lysine residues on histone proteins in order to regulate chromatin architecture and gene expression. These enzymes also post-translationally modify numerous non-histone targets, including transcription factors, chaperones and signaling molecules, resulting in changes in protein stability, protein-protein interactions, and protein-DNA interactions to control diverse cell functions (4). The family of conventional HDACs is comprised of 11 members that require $\mathrm{Zn}^{2+}$ as a cofactor for deacetylase activity, and are divided into four classes depending on their homology (5). Class I HDACs (HDAC1, 2, 3 and 8) generally localize to the nucleus, and class II HDACs (HDAC4, 5, 6, 7, 9 and 10) shuttle between the nucleus and cytoplasm. HDAC11, which shares similarities with both class I and II enzymes, is the sole member of the class IV group. The class III HDACs or SIRT1-7 require the cofactor nicotinamide adenine dinucleotide for activity, and act through a distinct mechanism which differs from class I, II and IV HDACs.

Small molecule inhibitors of HDACs, such as trichostatin A (TSA) and suberoylanilide hydroxamic acid (SAHA), have been pursued for their potential use as anticancer drugs (6). In addition to possessing anticancer activity, pan-HDAC inhibitors demonstrate effective anti-inflammatory properties, and their therapeutic effects have been demonstrated in septic shock, rheumatoid arthritis, multiple sclerosis and asthma (7-9). HDAC inhibitors (HDACis) have been found to exert antiviral effects; HDACis, such as TSA and SAHA, decrease hepatitis $\mathrm{C}$ virus (HCV) replication (10). However, the role of HDAC proteins in regulating paramyxovirus infections, particularly RSV infection, has not been previously reported, to the best of our knowledge.

In the present study, we aimed to explore the function of HDACis in RSV replication, and to determine the effect of HDACis on virus-related host defenses and inflammatory responses. Our results showed that RSV infection in the airway epithelium significantly increased HDAC2 expression, which is associated with aberrant histone acetylation. HDACis are capable of modulating innate antiviral responses and restricting RSV replication. Administering HDACis to RSV-infected mice may protect the animal against virus-induced lung injury. To the best of our knowledge, this is the first study to show that HDAC activity may affect the infectivity of RSV. This study has identified the important role of HDAC-related protein acetylation in the development of anti-RSV immunity.

\section{Materials and methods}

Chemicals and materials. TSA and SAHA were obtained from Sigma-Aldrich (St. Louis, MO, USA). Dulbecco's modified Eagle's medium (DMEM) and fetal bovine serum were purchased from Gibco-BRL, Life Technologies (Carlsbad, CA, USA). Malondialdehyde (MDA), nitric monoxide (NO), and myeloperoxidase (MPO) detection kits were purchased from Jiancheng Bioengineering Institute (Nanjing, China). Fluorescent-labeled anti-GR1 and anti-CD3 $\varepsilon$ MAb were purchased from BD Biosciences (San Jose, CA, USA). Human and mouse interleukin (IL)-6, tumor necrosis factor- $\alpha$ (TNF- $\alpha$ ) and IL-8/or KC enzyme-linked immunosorbent assay (ELISA) kits were purchased from Shanghai ExCell Biology Inc., (Shanghai, China) or eBioscience (San Diego, CA, USA). The antibodies against I $\mathrm{B} \alpha$ (44D4), total Stat3 (D3Z2G), phospho-Stat3 (Tyr705; D3A7), cyclo-oxygenase-2 (COX-2; D5H5), ERK (137F5), phospho-ERK (D13.14.4E), JNK, phospho-JNK (81E11), p-38 (D13E1), phospho-p-38 (D3F9), HDAC2 (3F3), acetyl-histone H3 (Ac-H3; C5B11), and GAPDH were purchased from Cell Signaling Technology, Inc. (Beverly, MA, USA).

Cell culture and treatment. The human bronchial epithelial cell line BEAS-2B was purchased from the American Type Culture Collection (ATCC; Rockville, MD, USA). The cells were cultured in DMEM supplemented with $10 \%$ (v/v) fetal calf serum (FCS), $2 \mathrm{mM}$ L-glutamine, $100 \mathrm{U} / \mathrm{ml}$ penicillin, and $100 \mathrm{mg} / \mathrm{ml}$ streptomycin (all from Life Technologies, Grand Island, NY, USA) (11). Plaque-purified human RSV-A2 (ATCC) and a transgenic RSV strain expressing green fluorescent protein (GFP-RSV), which was prepared as previously described (12), were grown in HEp-2 cells (ATCC). The transgenic RSV strain was used for a virus titration assay as a complementary method to the immunoplaque assay. BEAS-2B cells were plated at an appropriate density according to the experimental design for all experiments, and grown for $36 \mathrm{~h}$ prior to experimentation. Various doses (multiplicity of infection (MOI) $=5$ to 0.01 ) of RSV were used in our in vitro study, and RSV titers were determined using the immunoplaque assay according to previously described methods (13). The indicated concentrations of TSA or SAHA were added to the cultured BEAS-2B monolayer in order to determine the effect of HDAC2 inhibitors on cell viability. Cell viability was measured using a non-radioactive method (MTS-PMS assay) based on the formation of tetrazolium salts using a CellTiter 96 AQueous One Solution Cell Proliferation assay kit (Promega, Madison, WI, USA); the absorption rate was measured at $570 \mathrm{~nm}$ on a microplate reader (SpectraFluor; Tecan Austria Gmbh, Salzburg, Austria) (14). The cell viability assay was performed to determine the potential toxic effect of TSA or SAHA on the cultured airway epithelial cells. We found that treating BEAS-2B cells with $500 \mathrm{nM}$ TSA or SAHA did not affect cell viability, which suggests that the antiviral effect of TSA or SAHA is not due to a cytotoxic effect (data not shown).

Animal experiments. Eight to 10 week-old female BALB/c mice (sixty mice in total) were purchased from the Nanjing University Animal Research Center (Nanjing, China), and housed under specific pathogen free conditions (temperature, $25 \pm 1^{\circ} \mathrm{C}$; humidity, $60 \%$; and a 12 -h light cycle, 6:00 a.m. to 
Table I. Criteria for lung histopathological scoring.

\begin{tabular}{|c|c|c|c|c|}
\hline Score & Alveolar septae & Alveolar hemorrhage & Intra-alveolar fibrin & $\begin{array}{l}\text { Intra-alveolar } \\
\text { infiltrations/field }\end{array}$ \\
\hline 0 & All are thin and delicate & No hemorrhage & No intra-alveolar fibrin & $<5$ intra-alveolar cells \\
\hline 1 & $\begin{array}{l}\text { Congested alveolar septae } \\
\text { in }<1 / 3 \text { of the field }\end{array}$ & $\begin{array}{l}\text { Erythrocytes/alveolus } \\
\text { in } 1-5 \text { alveoli }\end{array}$ & $\begin{array}{l}\text { Fibrin strands in }<1 / 3 \\
\text { of the field }\end{array}$ & 5-10 intra-alveolar cells \\
\hline 2 & $\begin{array}{l}\text { Congested alveolar septae } \\
\text { in }<1 / 3-2 / 3 \text { of the field }\end{array}$ & $\begin{array}{l}\text { At least } 5 \text { erythrocytes/alveolus } \\
\text { in } 5-10 \text { alveoli }\end{array}$ & $\begin{array}{l}\text { Fibrin strands in } 1 / 3-2 / 3 \\
\text { of the field }\end{array}$ & 10-20 intra-alveolar cells \\
\hline 3 & $\begin{array}{l}\text { Congested alveolar septae } \\
>2 / 3 \text { of the field }\end{array}$ & $\begin{array}{l}\text { At least } 5 \text { erythrocytes/alveolus } \\
\text { in }>10 \text { alveoli }\end{array}$ & $\begin{array}{l}\text { Fibrin strands in }>2 / 3 \\
\text { of the field }\end{array}$ & $>20$ intra-alveolar cells \\
\hline
\end{tabular}

Table II. Primers used for qPCR validation of the transcriptional profiles.

\begin{tabular}{lll}
\hline & \multicolumn{2}{c}{ Primer sequences $\left(5^{\prime} \rightarrow 3^{\prime}\right)$} \\
\cline { 2 - 3 } Target gene & \multicolumn{1}{c}{ Forward } & \multicolumn{1}{c}{ Reverse } \\
\hline Murine- $\beta$-actin & GGCTGTATTCCCCTCCATCG & CCAGTTGGTAACAATGCCATGT \\
Human- $\beta$-actin & TGACGTGGACATCCGCAAAG & CTGGAAGGTGGACAGCGAGG \\
Murine-RSV-L & GAACTCAGTGTAGGTAGAATGTTTGCA & TTCAGCTATCATTTTCTCTGCCAAT \\
Human-RIG-I & GCCATTACACTGTGCTTGGAGA & CCAGTTGCAATATCCTCCACCA \\
Human-IFN- $\beta 1$ & TGCTCTGGCACAACAGGTAG & CAGGAGAGCAATTTGGAGGA \\
Murine-IFN- $\alpha$ & CCTTGACACTCCTGGTACAAATGA & GGATGGCTTGAGCCTTCTTG \\
Murine-IFN- $\beta 1$ & AGCTCCAAGAAAGGACGAACA & GCCCTGTAGGTGAGGTTGAT \\
\hline
\end{tabular}

IFN, interferon.

6.00 p.m.). The mice were given standard rodent chow, and allowed free access to water. All animal studies were performed in accordance with NIH guidelines, as described in the Guide for the Care and Use of Laboratory Animals of the NIH, and were approved by the Institutional Animal Care and Use Committee of Nanjing University (Nanjing, China). Ten mice from each group were infected, under light anesthesia with isoflurane, by intranasal inoculation of RSV $\left(5 \times 10^{5}\right.$ PFU) in $50 \mu 1$ phosphate-buffered saline (PBS) or sham infected with PBS as the control. RSV infection was confirmed by an ex vivo plaque assay according to a standard procedure described above. The HDAC2 inhibitors TSA $(0.5 \mathrm{mg} / \mathrm{kg} / \mathrm{mouse} /$ day $)$ and SAHA (25 $\mathrm{mg} / \mathrm{kg} / \mathrm{mouse} /$ day) were administered intraperitoneally for 3 days on days $-1,0$ and 1 of the experimental RSV infection period (15).

Histopathological analysis. At $48 \mathrm{~h}$ after RSV infection, half of the mice in each group were sacrificed with an injected overdose of pentobarbital; bronchoalveolar lavage fluid (BALF), serum, and lung tissues were collected. Levels of pro-inflammatory cytokines and oxidative stress enzymes were measured using the corresponding detection kits according to the manufacturer's instructions. For histopathological and immunohistochemical analyses, half of the mice in each group were sacrificed on day 4 post-RSV infection, and the lungs were lavaged by inserting a cannula into the trachea and instilling the lungs with $2 \times 0.5 \mathrm{ml}$ aliquots of ice-cold PBS. The lungs were subsequently fixed in $10 \%$ formalin, routinely processed and embedded in paraffin. Paraffin sections (4 $\mathrm{mm}$ each) were cut on glass slides. Hematoxylin and eosin (H\&E) staining and immunochemistry assay were performed according to standard protocols. Briefly, paraffin sections were blocked with $5 \%$ bovine serum albumin. The primary antibodies used were anti-I $\kappa \mathrm{B} \alpha \mathrm{Abs}$ or anti-COX-2 mAbs (Cell Signaling Technology, Inc.). Signals were captured using a PowerVision UltraVision Quanto Detection System (Thermo Scientific) according to the manufacturer's instructions. The results were examined under a light microscope (CKX31; Olympus, Tokyo, Japan) by a pathologist who was blinded to the group studies. The extent of the pathological lesions was graded from 0 to 3 (Table I). The score for each animal was calculated by dividing the total score for the number of observed sections (16).

The intensity of airway inflammation was assessed by measuring the total cell count and cell components in the BALF. The total cell counts in BALF were determined using a hemocytometer (XB.K.25; Shanghai Quijing Bohao Biotech Co., Ltd., Shanghai, China), and the individual cell components in the BALF were measured using cytospin staining or fluorescence-activated cell sorting (FACS), in order to distinguish the neutrophils from lymphocytes, based on the expression of GR-1 and CD3 on the cell surface (17). 
Measurement of viral replication and airway inflammation by reverse transcription-quantitative polymerase chain reaction (RT-qPCR). To determine the levels of RSV-induced cytokines and related interferon (INF)-I pathway signaling molecules, qPCR was performed. Total RNA from the cell culture and lung tissues was isolated using an RNA extraction kit purchased from BioTeke Corp., (Beijing, China). Total RNA was quantified, and $1 \mu \mathrm{g}$ of total RNA was reversed transcribed into cDNA and amplified using TaqMan Gene Expression Quantification assays (Applied Biosystems, Foster City, CA, USA). The cDNA was amplified and quantified using Applied Biosystems ${ }^{\circledR}$ StepOnePlus ${ }^{\mathrm{TM}}$ Real-Time PCR systems. Several sets of specific primers for RSV (RSV L gene) or INF-pathway-related signaling molecules, such as RIG-I, MDA-5, IFN- $\alpha$ and IFN- $\beta 1$, were designed. The sequences of primer pairs are listed in Table II.

qPCR analysis was performed using the comparative CT $(\triangle \mathrm{CT})$ method, in which $\mathrm{CT}$ is the threshold cycle number (the minimum number of cycles needed before the product can be detected). The amount of target genes was calibrated and normalized to an endogenous reference by the formula $2^{-\Delta \Delta \mathrm{CT}}$. The calculation of $2^{-\Delta \Delta \mathrm{CT}}$ provides a relative value when comparing the target with the calibrator, which was designated in this context as a fold increase of the RSV-infected group to the TSA or SAHA treatment group by relative quantification.

Western blot analysis. After treatment, BEAS-2B cells were harvested, washed with PBS, and lysed with RIPA cell lysis buffer containing $1 \%$ phenylmethylsulfonyl fluoride. The lysate was centrifuged for $15 \mathrm{~min}$ at $12,000 \mathrm{x} \mathrm{g}$ and $4^{\circ} \mathrm{C}$ to remove the insoluble cell fraction, and protein concentrations were measured by performing a bicinchoninic acid assay using a BCA kit (Pierce Chemical Co., Rockford, IL, USA). Equal amounts of proteins $(20 \mu \mathrm{g})$ from each sample were separated by $12 \%$ sodium dodecyl sulfate-polyacrylamide gel electrophoresis (SDS-PAGE), and transferred onto a nitrocellulose membrane (Millipore Corp., Billerica, MA, USA). Non-specific sites were blocked by incubating the membranes for $2 \mathrm{~h}$ at room temperature in $5 \%(\mathrm{w} / \mathrm{v})$ non-fat milk powder in Tris-buffered saline containing $0.05 \%$ Tween-20. The membranes were then incubated overnight at $4^{\circ} \mathrm{C}$ with primary and secondary HRP-conjugated antibodies. The membranes were visualized by enhanced chemiluminescence, and band intensity was measured and quantified.

Statistical analysis. Data represent at least three independent experiments, and are expressed as the means \pm SD. Statistical comparisons between different groups were measured using one-way ANOVA, followed by a Student's t-test. $\mathrm{P}<0.05$ was considered to indicate a statistically significant difference.

\section{Results}

$R S V$ infection alters histone acetylation levels by affecting HDAC2 expression. Emerging evidence highlights a critical function for HDACs and histone acetylation during viral infection (18); however, some viruses have evolved a wide array of mechanisms in order to subvert HDAC functions. To determine the effect of RSV infection on histone acetylation, we first examined the association between RSV infection and HDAC2 expression. Our results showed that RSV infection in
A
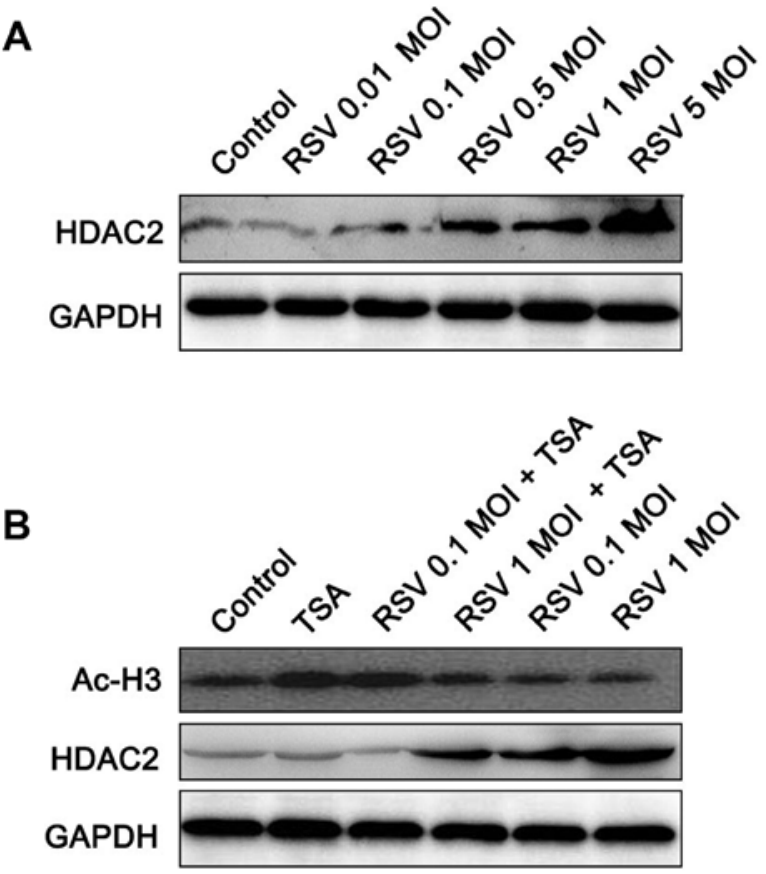

Figure 1. Respiratory syncytial virus (RSV) infection alters histone acetylation levels by affecting histone deacetylase 2 (HDAC2) expression. (A) BEAS-2B cells were infected with various doses of RSV $(\mathrm{MOI}=5,1.0,0.5,0.1,0.01)$ and the expression level of HDAC2 was assessed by western blot analysis. (B) Treatment of RSV-infected BEAS-2B cells [high dose $(\mathrm{MOI}=1)$ or low dose $(\mathrm{MOI}=0.1)$ ] with $500 \mathrm{nM}$ trichostatin A (TSA) for $24 \mathrm{~h}$ reversed the inhibition of RSV infection-induced histone acetylation.

BEAS-2B cells resulted in significantly increased expression of HDAC2 in a MOI-dependent manner (Fig. 1A). To analyze whether the induction of aberrant HDAC2 expression by RSV infection is the direct course of altered histone acetylation, TSA, a pan-HDAC inhibitor, was administered to the RSV-infected airway epithelial cells (AECs). Our results showed that the treatment of RSV-infected AECs with $500 \mathrm{~nm}$ TSA resulted in a significant decrease in HDAC2 expression, which inversely correlated with histone $\mathrm{H} 3$ acetylation (Fig. 1B). This result indicates that RSV-induced abnormal histone acetylation in AECs was due to the altered expression of HDAC2, and this effect was reversed by TSA treatment.

Inhibition of $R S V$ replication by HDACis. To examine the effect of HDAC2 expression on RSV replication, RSV-infected BEAS-2B cells were exposed to $500 \mathrm{nM}$ TSA for $24 \mathrm{~h}$, and RSV infection was assessed by a viral plaque assay. The treatment of RSV-infected BEAS-2B cells with TSA may significantly inhibit RSV infection, and this result was detected based on the number of virus-induced plaques formed (Fig. 2A). To further confirm this result, another selective HDAC2 inhibitor, SAHA, was administered to our system. The $\mathrm{IC}_{50}$ of TSA or SAHA for HDAC inhibition was 0.01-0.02 $\mu \mathrm{M}$, and treatment with TSA and SAHA at $1 \mu \mathrm{M}$ completely abolished the total HDAC activity. Our results show that the treatment of RSV-infected AECs with 500 nM SAHA significantly inhibited viral replication, which suggests that HDAC inhibition suppresses RSV replication.

HDAC2 affects type I IFN-dependent JAK/ signal transducer and activator of transcription (STAT) signaling and IFN-stimulated gene factor 3 (ISGF3) transcriptional 
A

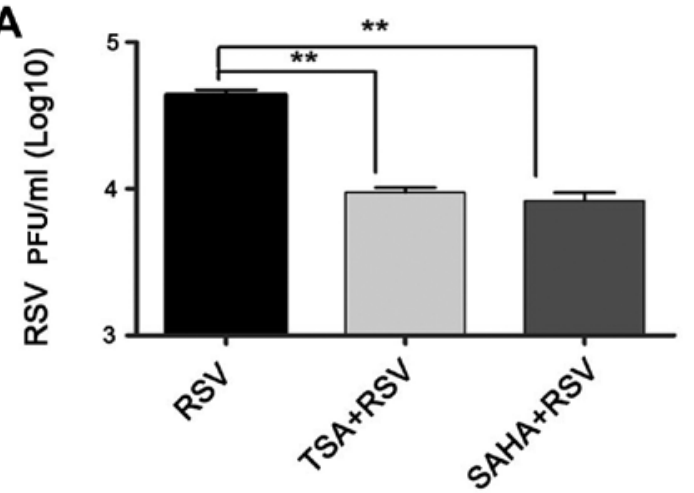

B
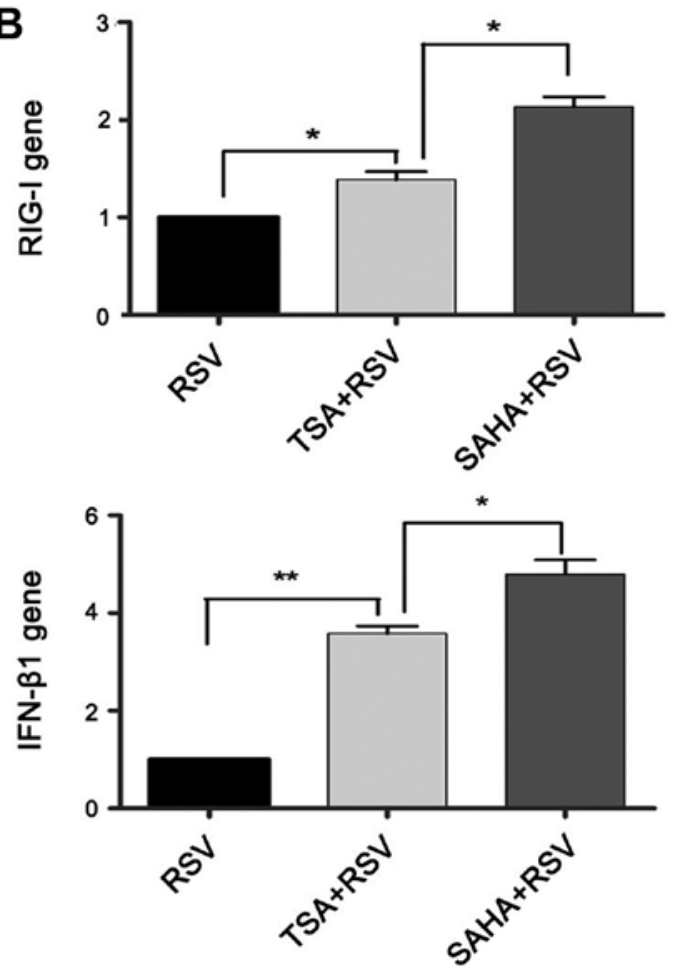

Figure 2. Treatment of respiratory syncytial virus (RSV)-infected airway epithelial cells (AECs) with trichostatin A (TSA) reduces RSV replication and upregulates the activation of the type I interferon (IFN) signaling pathway. (A) BEAS-2B cells were pre-incubated with $500 \mathrm{nM}$ TSA or suberoylanilide hydroxamic acid (SAHA) prior to the administration of RSV infection at an MOI of 0.5 . Viral replication was detected by the plaque assay using immunostaining with anti-RSV F protein MAbs; representative statistical results are shown $\left({ }^{* *} \mathrm{P}<0.01\right)$. (B) In vitro BEAS-2B cells were pre-treated with or without TSA or SAHA $(500 \mathrm{nM})$ for $1 \mathrm{~h}$. Cells were then stimulated with or without RSV (MOI=0.1) for an additional $1 \mathrm{~h}$. Levels of mRNA for RIG-I and IFN- $\beta 1$ were quantified by RT-qPCR. ("P<0.05 and $\left.{ }^{* *} \mathrm{P}<0.01\right)$.

activities (19). Therefore, the antiviral effect of HDAC2 inhibitors may be attributed to the activation of the type I IFN signaling pathway. To specifically examine the antiviral activity of HDAC2 inhibitors, we evaluated the activation status of the type I IFN signaling pathway during RSV infection together with HDAC2 inhibition. We found that the expression of type I IFN pathway-related molecules, such as RIG-I, significantly increased after RSV infection. The exposure of BEAS-2B cells to $500 \mathrm{nM}$ TSA significantly increased RIG-I gene expression. TSA exposure also enhanced the expression of IFN- $\beta 1$ (Fig. 2B), which was detected by qPCR. A trend for the increased expression of INF- $\alpha$ and MDA5 was also found, although the difference did not reach a significant level (data not shown). These results indicate that the IFN-I signaling pathway may be involved in the restriction of RSV replication.

Treatment of BEAS-2B cells with HDACis decreases $R S V$-induced inflammatory cytokine release and oxidative stress levels. HDAC is important in regulating various types of inflammation (7-9). To determine whether HDAC2 inhibitors affect RSV-induced airway inflammation, we measured inflammatory cytokine release in RSV-infected BEAS-2B cells exposed to TSA. As expected, we detected a considerable level of IL- 6 and IL- 8 release within $24 \mathrm{~h}$ following various doses of RSV (MOI=0.1, MOI=0.01) infection. However, TSA pre-treatment (500 nM) significantly inhibited both IL-6 and IL-8 production in the cell culture supernatants from RSV-infected BEAS-2B cells (Fig. 3A).

We also analyzed the levels of indicators of virus-induced oxidative stress. NO and MDA represent the membrane lipid oxidation levels in oxidative damage. RSV infection significantly increased the NO and MDA levels in the cell culture supernatants in an infection dose (MOI)-dependent manner (Fig. 3B). Treatment with the HDACi TSA significantly reduced the production of these oxidative molecules. Therefore, our results indicate that TSA exposure inhibited RSV-induced inflammatory cytokine release and oxidative injury. Given that TSA exhibited important anti-inflammatory effects, we measured the activation status of the related anti-inflammatory signaling pathways during RSV infection to further explore the mechanism. We observed that nuclear factor- $\kappa \mathrm{B}(\mathrm{NF}-\kappa \mathrm{B})$, Stat3, COX-2 and MAPK signaling pathways were all activated during the process of RSV infection. RSV infection significantly activated NF- $\kappa$ B, which was observed via I $\mathrm{B} \alpha$ degradation (Fig. $3 \mathrm{C}$ ). The increased activation of the Stat $3, \mathrm{COX}-2$ and MAPK signaling pathways was also detected in RSV-infected AECs; however, TSA treatment alleviated these effects. Notably, we observed that the phosphorylation level of p38 significantly increased in response to TSA treatment. In comparison, there were no significant changes in the expression of $\mathrm{p}$-ERK and $\mathrm{p}$-JNK following TSA treatment. This indicates that changes in the activation of p38, rather than JNK and ERK, may serve to alleviate the RSV-induced inflammation and restrict virus replication during RSV infection in airway epithelial cells. Therefore, our results indicate that HDCAi treatment reduces RSV-induced airway inflammation by activating the relevant signaling pathways.

HDACis protect against RSV infection in vivo. To assess the inhibitory effect of HDAC2 inhibitors on virus production in vivo, a mouse model of RSV-infection was established, and the antiviral effects of two HDACis (TSA and SAHA) were assayed. Our results showed that treatment with TSA or SAHA significantly inhibited RSV replication in vivo. The detection of viral replication using qPCR showed that exposing RSV-infected mice to HDACis (TSA or SAHA) significantly decreased viral load $(\mathrm{P}<0.05)$, and RSV L gene expression was inhibited by up to $75 \%$ in response to either TSA or SAHA exposure (Fig. 4A).

Pro-inflammatory cytokine production was measured using an ELISA, and the results showed that HDACi (TSA or SAHA) treatment also significantly downregulated pro-inflammatory cytokine release. High levels of pro-inflammatory cytokines, 
A

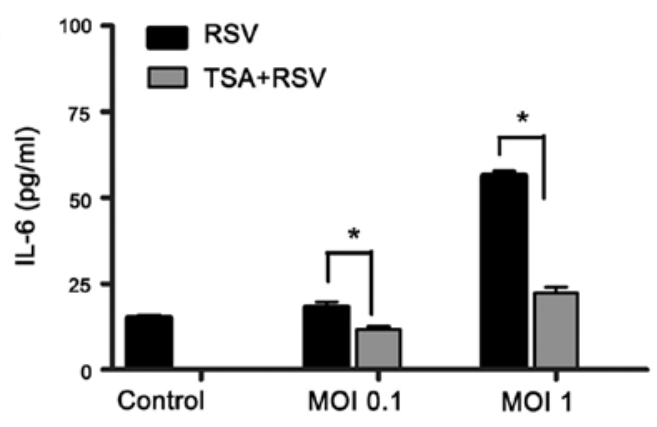

B

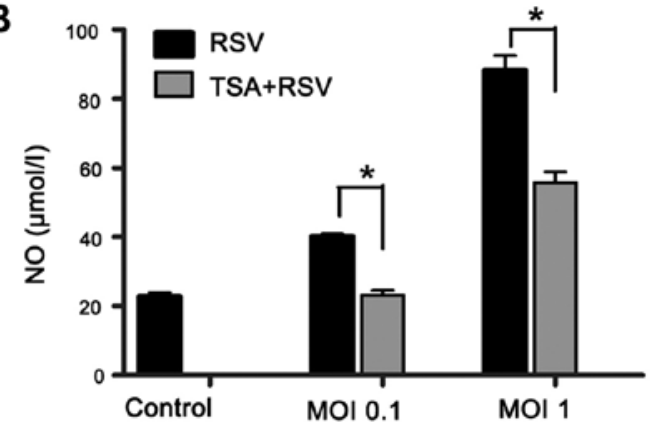

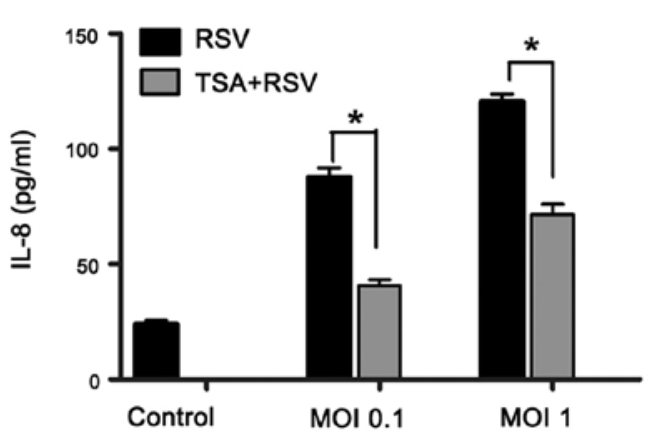

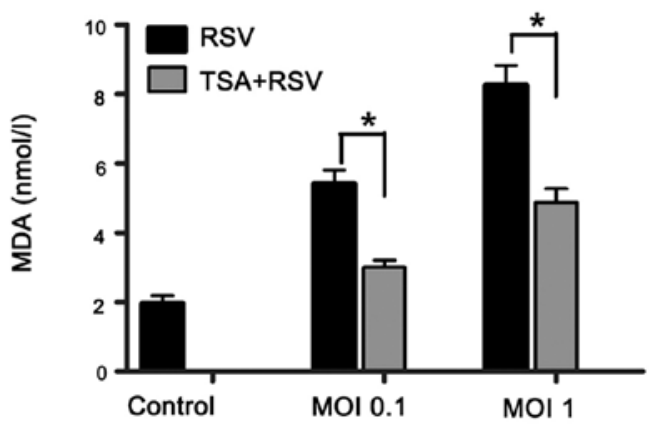

C

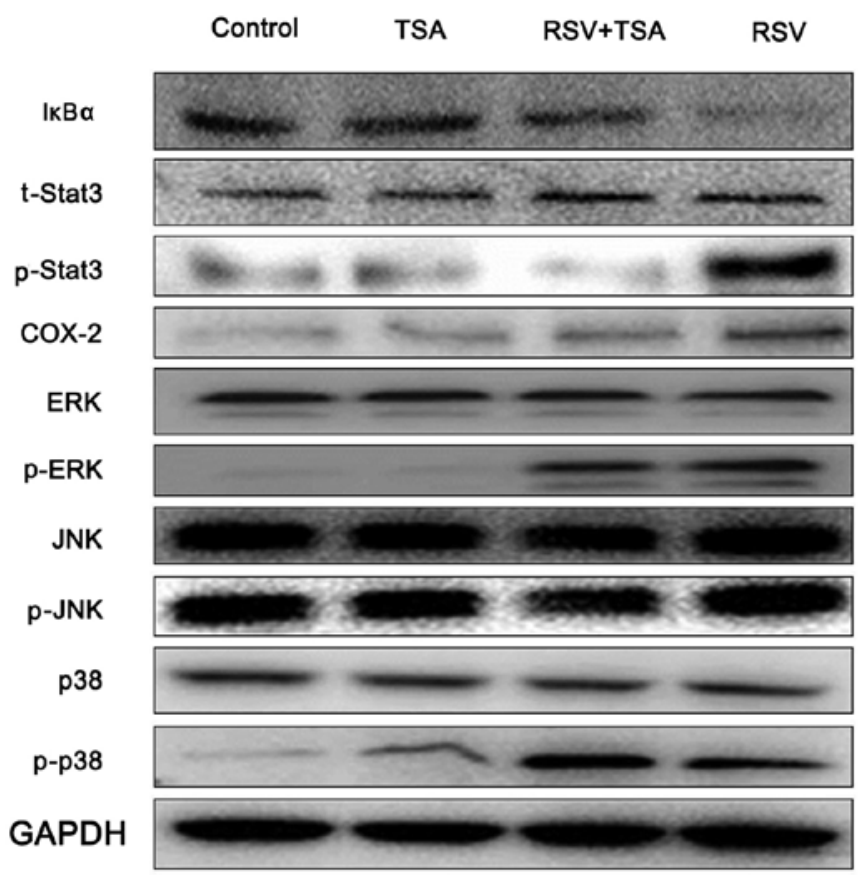

Figure 3. Treatment of respiratory syncytial virus (RSV)-infected airway epithelial cells (AECs) with trichostatin A (TSA) reduces pro-inflammatory cytokine production and decreases the release of oxidative stress-related molecules. (A) BEAS-2B cells were treated with 500 nM TSA, and infected with various doses of RSV (MOI=0.1 or 1). Cell supernatants were harvested at $24 \mathrm{~h}$ post-infection to measure the production of pro-inflammatory cytokines [interleukin (IL)-6 and IL-8], by ELISA. (B) Release of oxidative stress-related molecules [malondialdehyde (MDA) and nitrogen monoxide (NO)] were measured using specific kits. The results are expressed as the means $\pm \mathrm{SD}$; ${ }^{\mathrm{P}}<0.05$ compared with the untreated RSV-infected cells. (C) Cells were infected with RSV (MOI=0.1) for $1 \mathrm{~h}$ with or without TSA $(500 \mathrm{nM})$ treatment. Total lysates were prepared from each group using RIPA lysis buffer. The expression and phosphorylation of

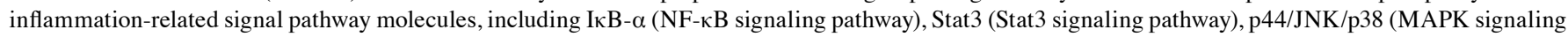
pathway), and COX-2, were assessed by western blot analysis; GAPDH was applied as an internal loading control.

namely IL- 6 and TNF- $\alpha$, were detected in the BALF $48 \mathrm{~h}$ after RSV infection (Fig. 4B). Both TSA and SAHA treatments reduced the release of pro-inflammatory cytokines. Airway and parenchymal lung inflammation was monitored in BALF cells and lung sections harvested on day 4 post-infection. Oxidative stress has been reported to be involved in RSV-induced lung injury (20), which was confirmed by our results, as evidenced by the increased release of neutrophil-related enzymes (MPO). 

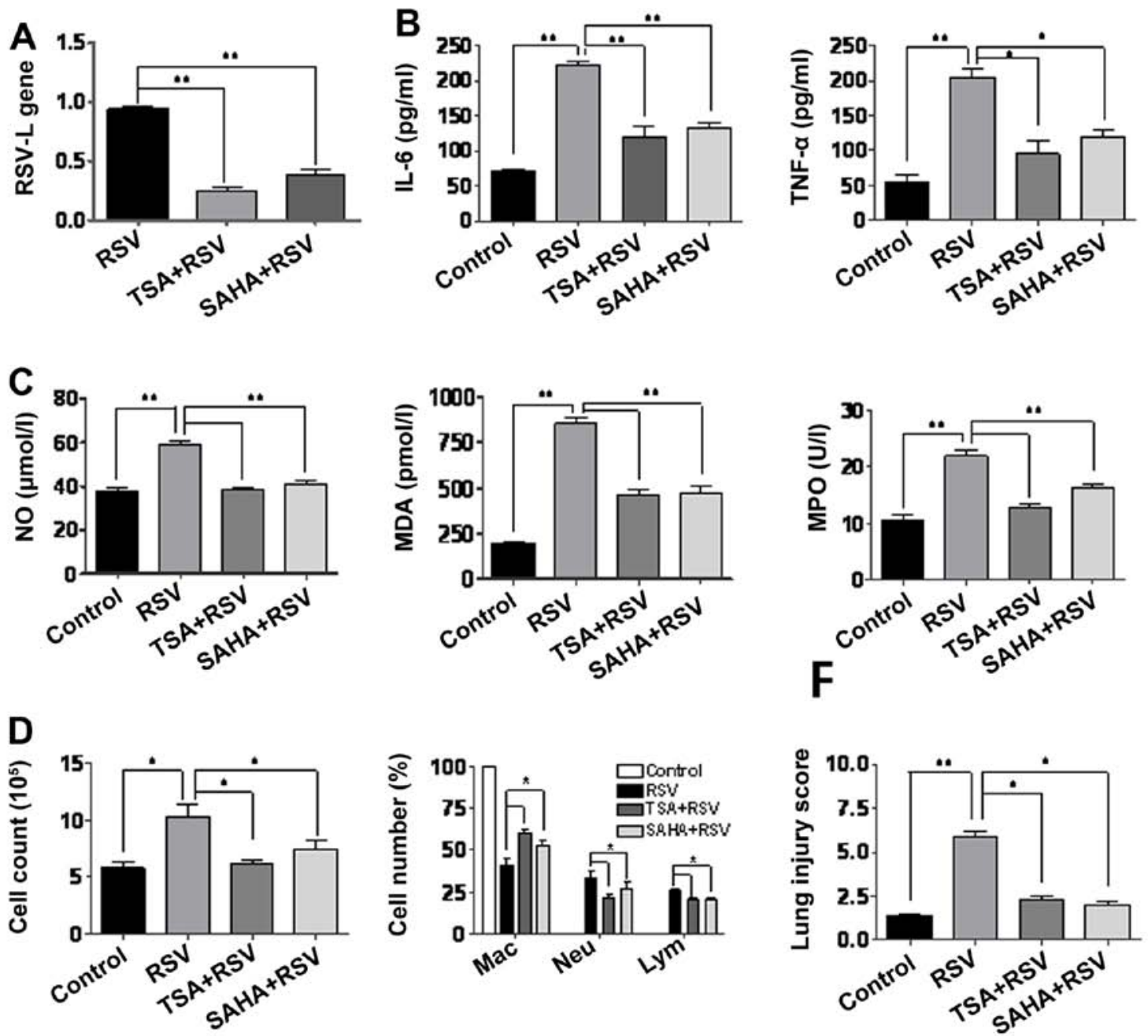

E

Control

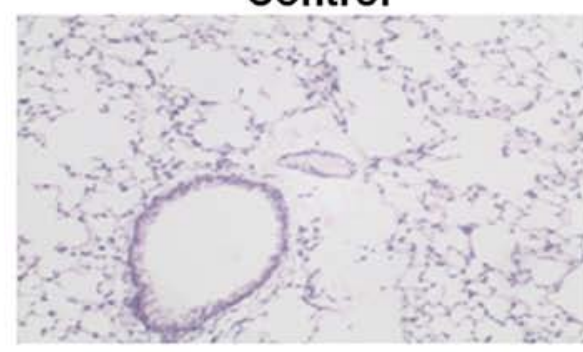

TSA+RSV

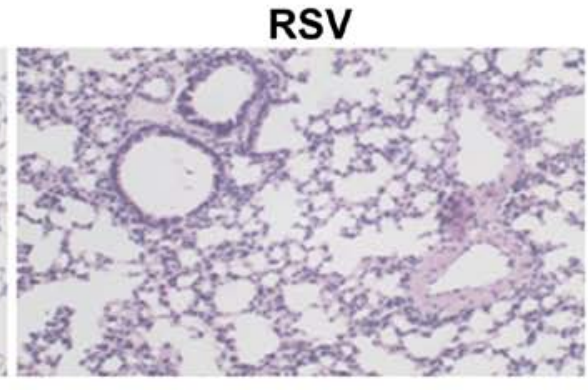

SAHA+RSV

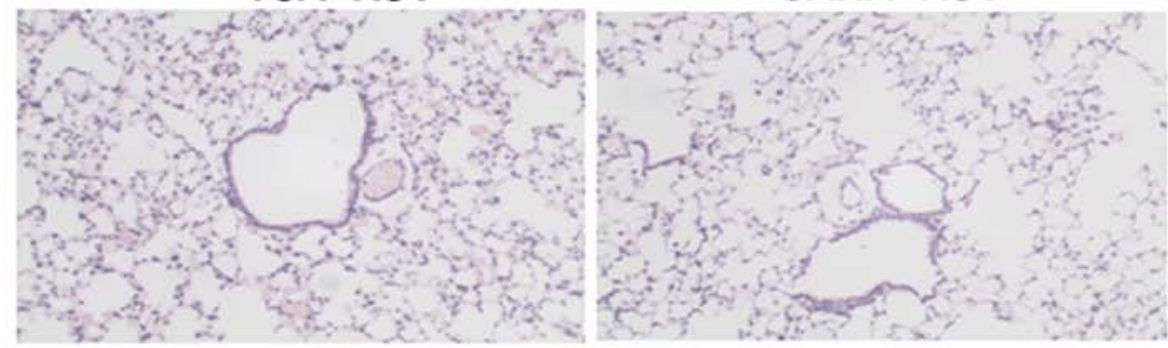

Figure 4. Treatment of respiratory syncytial virus (RSV)-infected mice with histone deacetylase inhibitors (HDACis) inhibits viral replication and reduces RSV-induced acute inflammation. BALB/C mice were exposed to trichostatin A (TSA) or suberoylanilide hydroxamic acid (SAHA) constitutively for 3 days. On the second day after treatment, mice were infected with $5 \times 10^{5} \mathrm{PFU}$ RSV. At $48 \mathrm{~h}$ post-infection, mice were sacrificed, and blood and bronchoalveolar lavage fluid (BALF) were collected, and the lungs were harvested. (A) The level of RSV replication in the lung tissues was quantified using RT-qPCR by measuring RSV-L gene expression. (B) Expression of pro-inflammatory cytokines in the BALF was measured by ELISA. (C) Production of oxidative stress-related molecules in the BALF [nitrogen oxide (NO), malondialdehyde (MDA) and myeloperoxidase (MPO)] were measured using specific detection kits. (D) Numbers of pro-inflammatory cells were assessed by cytospin staining and fluorescence-activated cell sorting (FACS). (E) Severity of airway inflammation and the histologic parameters of lung injury were assessed by H\&E staining of lung sections. (F) Average scores of airway inflammation and lung injury in each experimental group were determined and analyzed ( $\mathrm{P}<0.05$ and $\left.{ }^{* *} \mathrm{P}<0.01\right)$. Mac, macrophage; Neu, neutrophil; Lym, lymphocyte. 
High levels of NO and MDA production were also detected in the BALF after in vivo RSV infection. However, treatment with HDACis (TSA or SAHA) significantly reduced the levels of these oxidative stress-related factors, including MDA, NO and MPO (Fig. 4C). Analysis of BALF cells revealed an increased total cell count which was accompanied by a higher frequency of neutrophil and lymphocyte infiltration in the BALF. The administration of HDACis (TSA or SAHA) resulted in a significant decrease in inflammatory cell infiltration in the BALF, which was detected using a cytospin assay (Fig. 4D).

Scoring of histological sections stained with H\&E also revealed that airway and parenchymal inflammation following RSV infection was alleviated in the presence of HDACis (TSA or SAHA) with marked peribronchial, perivascular and alveolar infiltrates (Fig. 4E and F). Thus, these results demonstrate the profound anti-inflammatory effects of HDAC2 inhibitors during RSV infection of the lungs.

HDACis downregulate inflammatory-related signaling pathways and upregulate IFN-related signaling pathways during in vivo RSV infection. Our in vitro studies using cultured human epithelial cells demonstrated that a number of inflammatory signaling pathways were involved in RSV-induced lung injury. Thus, we performed another study in order to determine the mechanism by which HDAC inhibition protects mice against RSV infection in vivo. We observed that RSV infection induced the increased activation of $N F-\kappa B$ and COX-2 signaling $48 \mathrm{~h}$ after RSV infection, as evidenced by the decreased expression of I $\mathrm{B} \alpha$ (Fig. $5 \mathrm{~A}$ and $\mathrm{B}$ ) and the increased expression of COX-2 in the airway epithelium (Fig. 5C and D). However, these effects were blocked in response to treatment with the HDAC2 inhibitors TSA and SAHA.

The increased activation of the type I IFN pathway and the release of IFN- $\alpha$ and IFN- $\beta 1$, which were observed in our in vitro study, were also demonstrated in our in vivo study. Treatment with HDACis (TSA and SAHA) essentially enhanced the gene expression levels of IFN- $\alpha$ and IFN- $\beta 1$ (Fig. 5E). This result confirms that activation of the IFN-I signaling pathway may be a crucial antiviral mechanism mediated by HDACis.

\section{Discussion}

Despite intensive research, the pathological mechanisms underlying RSV infection remain unclear. One of the crucial points is that individuals present with different clinical symptoms after RSV infection; some have only mild cold-like signs and symptoms and require no specific treatment whereas in others, RSV infections may lead to more serious illnesses, including bronchiolitis or pneumonia, which are potentially fatal and require hospitalization (21). Apart from genetic variations, the environmental factors, particularly the epigenetic mechanisms that may influence viral replication and tissue injury, need to be defined in order to explain these different reactions following $\mathrm{RSV}$ infection.

Histone acetyltransferases and HDACs play crucial roles in the regulation of gene expression as they regulate the chromatin structure by acetylating and deacetylating histones, respectively; as prominent modulators of histone acetylation, HDACs are essential transcriptional and epigenetic regulators (22). A previous study highlighted the critical function of histone acetylation in controlling viral infection (23). In the present study, we attempted to determine whether HDAC activity and histone acetylation play a role in the regulation of RSV replication and virus-induced airway inflammation.

In the lungs, HDAC2 is crucial for the development of airway inflammation. The aberrant expression of HDAC2 appears to mediate the action of steroidal resistance in patients with severe asthma or chronic obstructive pulmonary disease (COPD) (24). Given that severe RSV bronchiolitis has been associated with an susceptibility to the subsequent development of asthma and exacerbation of COPD, we first analyzed the effect of RSV infection on HDAC2 expression. We demonstrated that HDAC2 expression was profoundly increased, and histone $\mathrm{H} 3$ acetylation was downregulated after RSV infection. The treatment of RSV-infected AECs with the HDACi TSA completely reversed these effects. Simultaneously, significantly reduced viral replication was observed in response to TSA exposure. Taken together, these findings suggest that the induction of upregulated HDAC2 expression by RSV may be an important mechanism which facilitates viral replication, whereas the enhanced level of histone acetylation may suppress viral replication.

HDACs have been associated with viral replication and pathogenesis during infection with a number of human pathogens, including herpesviruses, human papilloma virus, coxsackievirus, hepatitis $\mathrm{B}, \mathrm{HCV}$ and human immunodeficiency virus (HIV)-1 (18). During coxsackievirus infection, HDACis suppressed coxsackievirus B3 growth in vitro and myocarditis-induced growth in mice (25). During HCV infection, the HDACi SAHA exerts a suppressive effect on HCV replication by altering the expression of genes, such as OPN and Apo-A1, in host cells (10). Similar results were found for other viral infections, such as hepatitis B (26). There is a correlation between HDAC activity and the establishment of latent viral infection (27). Different HDAC-controlled chromatin remodeling and acetylation/deacetylation events have been asssociated with the regulation of promoter activity and the maintenance of viral latency during herpesvirus infection. Similarly, HDACis have been used to activate HIV transcription in latently infected cells, and eradicate latent HIV infection (28). Based on existing evidence, HDACis may be used as novel therapeutic tools in the management of several types of viral infections. These findings rely on various mechanisms. Firstly, HDACis are capable of reactivating the latent virus present in infected cells, and of favoring the clearance of the virus from cellular reservoirs, such as in HIV and human cytomegalovirus (HCMV) infections. Secondly, HDACis favor the apoptosis of infected cells, leading to the elimination of the infected cells. This mechanism may potentially be used to kill transformed cells infected by viruses, such as human papillomavirus and Epstein-Barr virus.

In the present study, we also examined the correlation between HDAC2 expression and anti-RSV host defenses to determine the effect of histone acetylation on RSV infection. IFN- $\alpha / \beta$ signaling is crucial for combating viral infection; however, this signaling pathway is under tight control. HDACs may affect the activation of IFN- $\alpha / \beta$ signaling. However, based on previous studies, the regulatory function of HDACs affects multiple aspects of IFN- $\alpha / \beta$ signaling. On the one hand, HDACs 


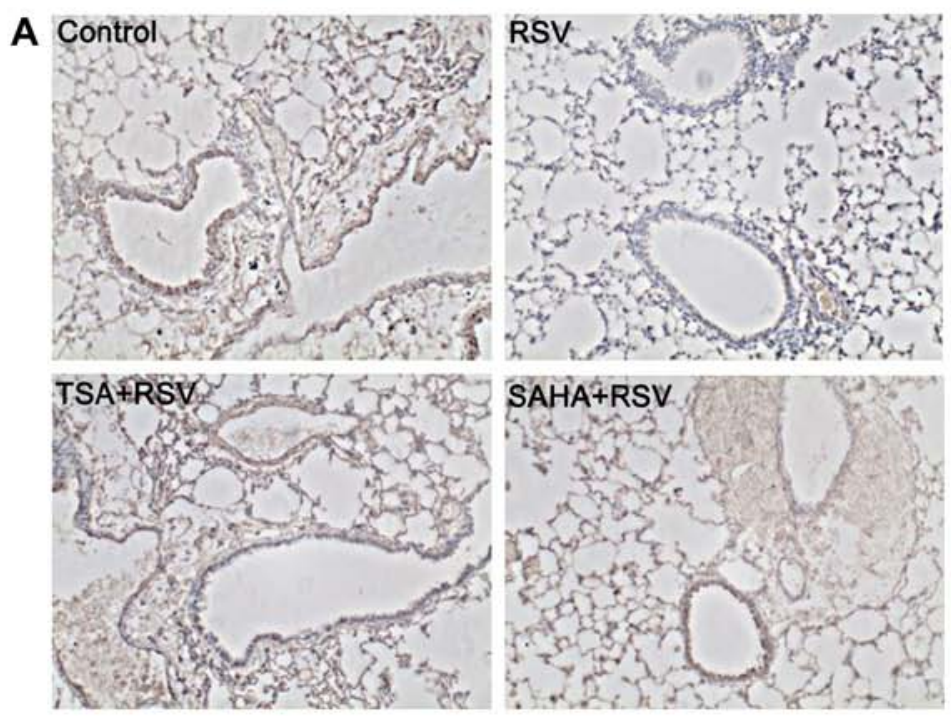

\section{B}

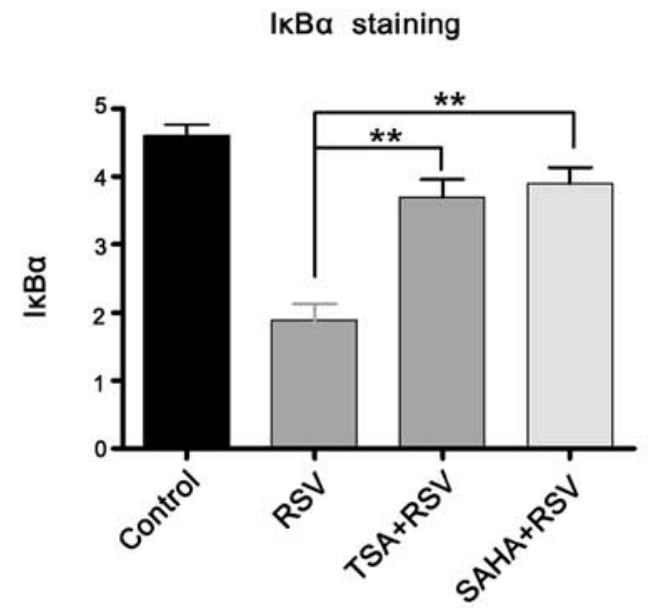

D
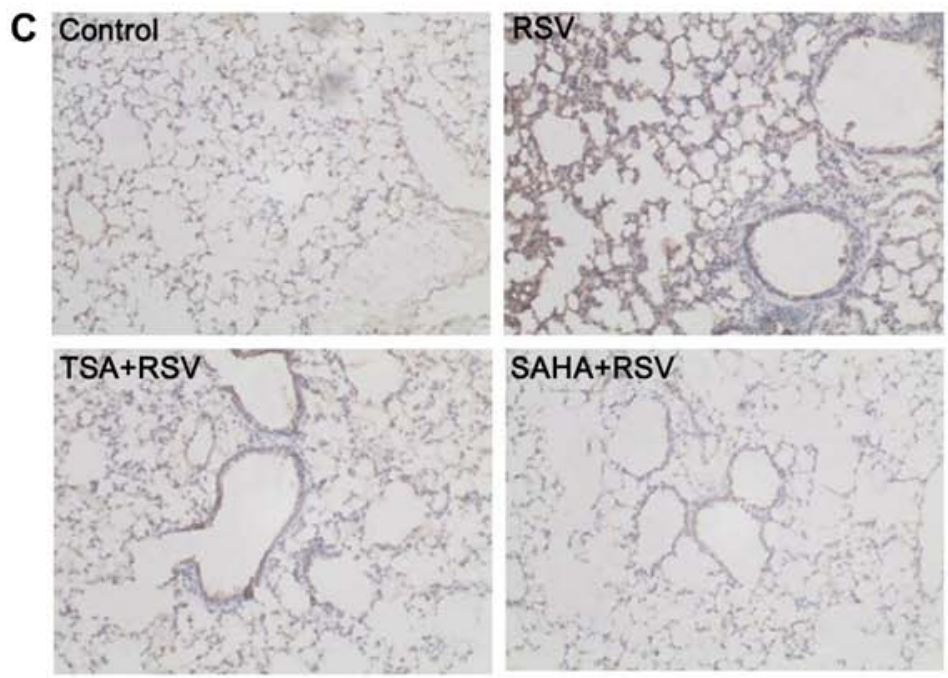

SAHA+RSV

$\mathbf{E}$

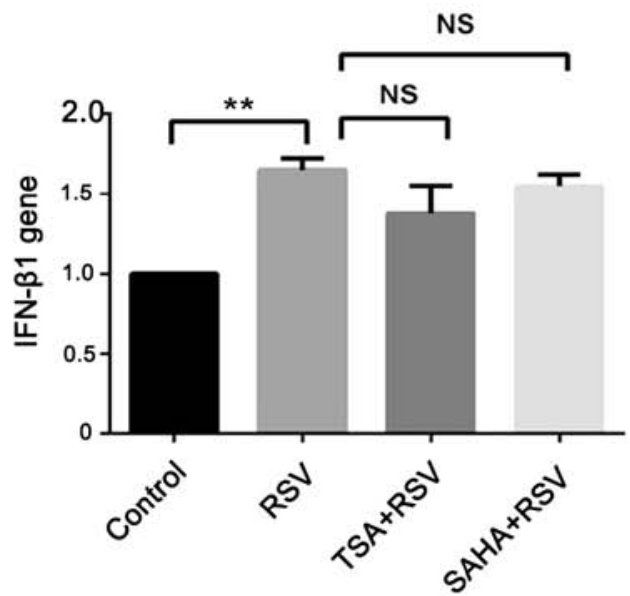

Figure 5. Histone deacetylase inhibitor (HDACi) exposure enhances the activation of interferon (IFN)-I-related signal pathways and decreases the activation of nuclear factor- $\mathrm{KB}(\mathrm{NF}-\kappa \mathrm{B})$ and $\mathrm{COX}-2$ signaling pathways in a mouse model of respiratory syncytial virus (RSV) infection. (A and C) Immunohistochemistry staining of inflammation-related signaling pathways in the mouse lung sections. Mouse lungs were harvested at day 2 post-RSV infection. Lung section slides were stained with anti-IкB $\alpha$ Abs or anti-COX-2 Abs, and goat IgG was applied as a negative control (images not shown). The staining result was visualized by microscopy (magnification, x100); (B and D) Average score of IкB $\alpha$ and COX-2 positive staining from the immunohistochemistry assay. (E) Mouse lungs were harvested at day 2 post-RSV infection, and total RNA samples were prepared to measure IFN- $\alpha /$ $\beta 1$ gene transcription levels by qPCR. Data are representative of at least two independent experiments with similar results. ${ }^{*} \mathrm{P}<0.05$ and ${ }^{* *} \mathrm{P}<0.01$ were considered to indicate a statistically significant difference. 
alter the expression of IFN- $\alpha / \beta$; histone acetylation correlates with virus infectivity, and viral infection leads to a marked hyperacetylation of histones $\mathrm{H} 3$ and $\mathrm{H} 4$, which are localized to the IFN- $\beta$ promoter. On the other hand, HDACs may alter cell sensitivity to IFN; HDACs play an essential role in the transcriptional induction of IFN-stimulated genes (ISGs). The inhibition of HDAC function leads to a significant impairment of ISG expression (29), demonstrating that HDACs may be required for ISG transcription. Apart from type I IFNs, type III IFNs (IFN- $\lambda \mathrm{s}$ ) are also induced upon viral infection, activate ISG expression, and display broad-spectrum antiviral activity. IFNLR1 expression may be epigenetically silenced by HDAC, and HDACis are capable of increasing receptor expression and restoring sensitivity to IFN- $\lambda$ in previously non-responsive cells, thereby enhancing protection against viral pathogens (30).

Broad-acting inhibitors of HDAC exert anti-inflammatory effects both in vitro and in vivo. HDACis, such as TSA, reduced inflammation in a murine model of allergic asthma (31). A similar study of lipopolysaccharide-induced acute murine lung injury was performed using the HDACis butyrate (32). The decreased expression of HDAC2 has been observed in a patient with COPD compared with that in a healthy control individual (33). To elucidate the varied functions of HDAC2 in regulating airway inflammation, both in vivo and in vitro approaches were used in order to examine the regulatory functions of HDAC2is in controlling RSV-induced airway inflammation. The present study demonstrated that using TSA, or even the more specific HDAC2 inhibitor SAHA, significantly alleviated RSV-induced pro-inflammatory cytokine production, including IL-6 and IL-8. Given that the increased expression of IL-6 correlated with a decrease in Treg function, which may explain the correlation between RSV infection and asthma sensitization, and IL-8 is the major cytokine known to mediate neutrophil chemotaxis in the lungs (34), the reduced expression of IL-6 and IL-8 may explain why HDACis exposure decreased neutrophil and $\mathrm{T}$ cell infiltration in a mouse model of RSV infection. We also observed that administering HDACis significantly decreased the activation of inflammatory signaling pathways, namely NK- $\kappa \mathrm{B}, \mathrm{MAPKs}, \mathrm{COX}-2$ and Stat3. These results further demonstrate the key role of HDACis treatment in regulating RSV-induced airway inflammation by mediating the activation of the related signaling pathways.

Oxidative stress is important in the pathogenesis of RSV bronchiolitis. Oxidative stress-related factors may be produced by AECs and airway endothelial cells that are involved in many forms of tissue damage, including damage to cellular components, such as lipids, proteins and DNA (35). A previous study showed that RSV infection of AECs induced reactive oxygen species (ROS) production, which is involved in transcription factor IRF gene activation and chemokine gene expression (36); the decreased expression and activity of antioxidant enzymes may contribute to this effect. In the present study, we observed the significantly increased production of NO, MDA and MPO in RSV-infected AECs, as well as in the lungs of RSV-infected mice. Taken together, these findings indicate that HDACi treatment significantly ameliorates RSV-induced oxidative stress, clinical disease and pulmonary inflammation in a mouse model of RSV infection, suggesting a causal relationship among HDAC activity, ROS production and lung injury.
In conclusion, this study identified novel functions of HDACis in controlling RSV infection and regulating virus-induced airway inflammation. Our data indicate that RSV infection altered histone acetylation by affecting HDAC2 expression. HDACi treatment may restrict RSV replication by activating the type I IFN signaling pathway. RSV-induced airway inflammation was prevented by the administration of HDACis which suppressed the release of pro-inflammatory cytokines and decreased ROS production. These dual effects of HDACis provide insights into the host antiviral defense mechanism, and suggest important molecular targets for establishing a novel therapeutic strategy for preventing RSV infection.

\section{Acknowledgements}

The present study was supported by grants from the National Natural Science Foundation of China (nos. 81471095 and 91543131), the Science and Technology Research Programs of Health and Family Planning Commission of Jiangsu Province (no. H201505), the Research Projects in Traditional Chinese Medicine Industry of China (no. 201507004-2), and the National High Technology Research and Development Program ('863' Program) of China (no. 2014AA022204).

\section{References}

1. Shay DK, Holman RC, Newman RD, Liu LL, Stout JW and Anderson LJ: Bronchiolitis-associated hospitalizations among US children, 1980-1996. JAMA 282: 1440-1446, 1999.

2. Monick M, Staber J, Thomas K and Hunninghake G: Respiratory syncytial virus infection results in activation of multiple protein kinase $\mathrm{C}$ isoforms leading to activation of mitogen-activated protein kinase. J Immunol 166: 2681-2687, 2001.

3. Rudd BD, Burstein E, Duckett CS, Li X and Lukacs NW: Differential role for TLR3 in respiratory syncytial virus-induced chemokine expression. J Virol 79: 3350-3357, 2005.

4. Minucci S and Pelicci PG: Histone deacetylase inhibitors and the promise of epigenetic (and more) treatments for cancer. Nat Rev Cancer 6: 38-51, 2006.

5. de Ruijter AJM, van Gennip AH, Caron HN, Kemp S and van Kuilenburg ABP: Histone deacetylases (HDACs): characterization of the classical HDAC family. Biochem J 370: 737-749, 2003.

6. Lane AA and Chabner BA: Histone deacetylase inhibitors in cancer therapy. J Clin Oncol 27: 5459-5468, 2009.

7. Grabiec AM, Krausz S, de Jager W, Burakowski T, Groot D, Sanders ME, Prakken BJ, Maslinski W, Eldering E, Tak PP and Reedquist KA: Histone deacetylase inhibitors suppress inflammatory activation of rheumatoid arthritis patient synovial macrophages and tissue. J Immunol 184: 2718-2728, 2010.

8. Royce SG and Karagiannis TC: Histone deacetylases and their inhibitors: new implications for asthma and chronic respiratory conditions. Curr Opin Allergy Clin Immunol 14: 44-48, 2014.

9. Licciardi PV, Ververis K, Tang ML, El-Osta A and Karagiannis TC. Immunomodulatory effects of histone deacetylase inhibitors. Curr Mol Med 13: 640-647, 2013.

10. Miura K, Taura K, Kodama Y, Schnabl B and Brenner DA: Hepatitis $C$ virus-induced oxidative stress suppresses hepcidin expression through increased histone deacetylase activity. Hepatology 48: 1420-1429, 2008.

11. Wang M, Sun GB, Sun X, Wang HW, Meng XB, Qin M, Sun J, Luo Y and Sun XB: Cardioprotective effect of salvianolic acid B against arsenic trioxide-induced injury in cardiac H9c2 cells via the PI3K/Akt signal pathway. Toxicol Lett 216: 100-107, 2013.

12. Hallak LK, Spillmann D, Collins PL and Peeples ME: Glycosaminoglycan sulfation requirements for respiratory syncytial virus infection. J Virol 74: 10508-10513, 2000.

13. Wang H, Peters $\mathrm{N}$ and Schwarze J: Plasmacytoid dendritic cells limit viral replication, pulmonary inflammation, and airway hyperresponsiveness in respiratory syncytial virus infection. J Immunol 177: 6263-6270, 2006. 
14. Sun GB, Sun X, Wang M, Ye JX, Si JY, Xu HB, Meng XB, Qin M, Sun J, Wang HW and Sun XB: Oxidative stress suppression by luteolin-induced heme oxygenase-1 expression. Toxicol Appl Pharmacol 265: 229-240, 2012.

15. Cabanski M WJ, Zaslona Z, Steinmüller M, Fink L, Seeger W, Lohmeyer J: Genome-wide transcriptional profiling of mononuclear phagocytes recruited to mouse lungs in response to alveolar challenge with the TLR2 agonist Pam3CSK4. Am J Physiol Lung Cell Mol Physiol 297: L608-L618, 2009.

16. Matute-Bello G, Winn RK, Jonas M, Chi EY, Martin TR and Liles WC: Fas (CD95) induces alveolar epithelial cell apoptosis in vivo: implications for acute pulmonary inflammation. Am J Pathol 158: 153-161, 2001.

17. Belperio JA, Keane MP, Burdick MD, Londhe V, Xue YY, Li K, Phillips RJ and Strieter RM: Critical role for CXCR2 and CXCR2 ligands during the pathogenesis of ventilator-induced lung injury. J Clin Invest 110: 1703-1716, 2002.

18. Guise AJ, Budayeva HG, Diner BA and Cristea IM: Histone deacetylases in herpes virus replication and virus-stimulated host defense. Viruses 5: 1607-1632, 2013.

19. Icardi L, Lievens S, Mori R, Piessevaux J, De Cauwer L, De Bosscher K and Tavernier J: Opposed regulation of type I IFN-induced STAT3 and ISGF3 transcriptional activities by histone deacetylases (HDACS) 1 and 2. FASEB J 26: 240-249, 2012.

20. Garofalo RP, Kolli D and Casola A: Respiratory syncytial virus infection: mechanisms of redox control and novel therapeutic opportunities. Antioxid Redox Signal 18: 186-217, 2013.

21. Hall CB, Geiman JM, Biggar R, Kotok DI, Hogan PM and Douglas GR Jr: Respiratory syncytial virus infections within families. N Engl J Med 294: 414-419, 1976.

22. Peserico A and Simone C: Physical and functional HAT/HDAC interplay regulates protein acetylation balance. J Biomed Biotechnol 2011: 371832, 2011

23. Yagi K, Ishii M, Namkoong H, Fujii H, Asami T, Suzuki S, Asakura T, Mizoguchi K, Kamo T, Tasaka S, et al: Histone deacetylase inhibition protects mice against lethal postinfluenza pneumococcal infection. Crit Care Med: Jule 27, 2016 (Epub ahead of print)

24. Barnes PJ: Role of HDAC2 in the pathophysiology of COPD. Annu Rev Physiol 71: 451-464, 2009.

25. Zhou L, He X, Gao B and Xiong S: Inhibition of histone deacetylase activity aggravates Coxsackievirus B3-induced myocarditis by promoting viral replication and myocardial apoptosis. J Virol 89: 10512-10523, 2015.

26. Wang YCYX, Yang X, Xing LH and Kong WZ: Effects of SAHA on proliferation and apoptosis of hepatocellular carcinoma cells and hepatitis B virus replication. World J Gastroenterol 19 : $5159-5164,2013$
27. Roizman B: The checkpoints of viral gene expression in productive and latent infection: The role of the HDAC/CoREST/ LSD1/REST repressor complex. J Virol 85: 7474-7482, 2011.

28. Archin NM, Liberty AL, Kashuba AD, Choudhary SK, Kuruc JD, Crooks AM, Parker DC, Anderson EM, Kearney MF, Strain MC, et al: Administration of vorinostat disrupts HIV-1 latency in patients on antiretroviral therapy. Nature 487: 482-485, 2012.

29. Chang H-M, Paulson M, Holko M, Rice CM, Williams BR, Marié I and Levy DE: Induction of interferon-stimulated gene expression and antiviral responses require protein deacetylase activity. Proc Natl Acad Sci USA 101: 9578-9583, 2004.

30. Ding S, Khoury-Hanold W, Iwasaki A and Robek MD: Epigenetic reprogramming of the type III interferon response potentiates antiviral activity and suppresses tumor growth. PLoS Biol 12: e1001758, 2014.

31. Toki S, Goleniewska K, Reiss S, Zhou W, Newcomb DC, Bloodworth MH, Stier MT, Boyd KL, Polosukhin VV, Subramaniam S and Peebles RS Jr: The histone deacetylase inhibitor trichostatin A suppresses murine innate allergic inflammation by blocking group 2 innate lymphoid cell (ILC2) activation. Thorax 71: 633-645, 2016.

32. Ni YF, Wang J, Yan XL, Tian F, Zhao JB, Wang YJ and Jiang T: Histone deacetylase inhibitor, butyrate, attenuates lipopolysaccharide-induced acute lung injury in mice.Respir Res 11: 33, 2010.

33. Tan C, Xuan L, Cao S, Yu G, Hou Q and Wang H: Decreased histone deacetylase 2 (HDAC2) in peripheral blood monocytes (PBMCs) of COPD patients. PLoS One 11: e0147380, 2016.

34. Cacalano G, Lee J, Kikly K, Ryan AM, Pitts-Meek S, Hultgren B, Wood WI and Moore MW: Neutrophil and B cell expansion in mice that lack the murine IL-8 receptor homolog. Science 265: 682-684, 1994

35. Hosakote YM, Komaravelli N, Mautemps N, Liu T, Garofalo RP and Casola A: Antioxidant mimetics modulate oxidative stress and cellular signaling in airway epithelial cells infected with respiratory syncytial virus. Am J Physiol Lung Cell Mol Physiol 303: L991-L1000, 2012.

36. Jamaluddin M, Tian B, Boldogh I, Garofalo RP and Brasier AR: Respiratory syncytial virus infection induces a reactive oxygen species-MSK1-phospho-Ser-276 RelA pathway required for cytokine expression. J Virol 83: 10605-10615, 2009. 